\title{
GeneWeaver: finding consilience in heterogeneous cross-species functional genomics data
}

\author{
Jason A. Bubier ${ }^{1}$ - Charles A. Phillips ${ }^{2}$ - Michael A. Langston ${ }^{2}$ Erich J. Baker ${ }^{3}$. \\ Elissa J. Chesler ${ }^{1}$
}

Received: 21 April 2015/ Accepted: 3 June 2015/Published online: 20 June 2015

(C) The Author(s) 2015. This article is published with open access at Springerlink.com

\begin{abstract}
A persistent challenge lies in the interpretation of consensus and discord from functional genomics experimentation. Harmonizing and analyzing this data will enable investigators to discover relations of many genes to many diseases, and from many phenotypes and experimental paradigms to many diseases through their genomic substrates. The GeneWeaver.org system provides a platform for cross-species integration and interrogation of heterogeneous curated and experimentally derived functional genomics data. GeneWeaver enables researchers to store, share, analyze, and compare results of their own genome-wide functional genomics experiments in an environment containing rich companion data obtained from major curated repositories, including the Mouse Genome Database and other model organism databases, along with derived data from highly specialized resources, publications, and user submissions. The data, largely consisting of gene sets and putative biological networks, are mapped onto one another through gene identifiers and homology across species. A versatile suite of interactive tools enables investigators to perform a variety of set analysis operations to find consilience among these often noisy experimental results. Fast algorithms enable real-time analysis of large queries. Specific applications include prioritizing candidate genes for quantitative trait loci, identifying biologically valid mouse models and phenotypic assays for human
\end{abstract}

Elissa J. Chesler

Elissa.Chesler@jax.org

The Jackson Laboratory, Bar Harbor, ME 04609, USA

2 Department of Electrical Engineering and Computer Science, University of Tennessee, Knoxville, TN 37996, USA

3 Computer Science Department, Baylor University, Waco, TX 76798, USA disease, finding the common biological substrates of related diseases, classifying experiments and the biological concepts they represent from empirical data, and applying patterns of genomic evidence to implicate novel genes in disease. These results illustrate an alternative to strict emphasis on replicability, whereby researchers classify experimental results to identify the conditions that lead to their similarity.

\section{Heterogeneous functional genomics data integration in GeneWeaver}

There is a growing need to find consensus among large collections of diverse biological data to relate molecular mechanisms to disease and to understand the relations among diverse disease characteristics, models, and mechanisms through their underlying biology. Researchers have an ever-expanding set of technologies for the characterization and quantification of genetic variants, gene products, and other biological molecules associated with disease. This genome-wide experimentation has produced a substantial quantity of diverse data on the associations among genetic variants, model organism phenotypes, gene expression patterns, and many other disease-related endpoints. Typically, the outcomes of functional experiments consist of a set of genomic features, often genes or transcripts, but increasingly other entities such as allelic variants, methylation sites, small non-coding RNA, and an expanding variety of new biomolecular endpoints. Each of these is associated with a biological or behavioral assay, which is in turn associated with a disease-related construct. Often, the evidences for such associations vary across species and experimental paradigms. This variation can help unravel relationships among biological processes and 
diseases, facilitate an improved characterization of disease, identify precision animal models of human disease, and refine classification of ambiguous diseases. Ultimately, these developments will lead to refined recognition of biological mechanisms, diagnostics, and therapeutics through detection of conserved roles of genes in multiple biological contexts.

The GeneWeaver software system (Baker et al. 2012) contains a database and a suite of tools to enable dissemination, integration, analysis, and discovery from heterogeneous data derived through functional genomics experimentation and biological data curation (Fig. 1). There are many paths of inquiry made possible with heterogeneous functional genomics data, each aimed at discovery of convergent evidence for gene-gene, gene-disease, or disease-disease relations. As data from gene expression and genetic mapping studies accumulate, investigators have realized the potential for statistical meta-analysis to refine presumptive associations of genetic loci and disease. Recent efforts aim to discover the differences among studies in which the effects occur (Kang et al. 2014). Most collections of functional genomics data sets related to a specific biological or behavioral concept are highly heterogeneous, however, and therefore are not amenable to quantitative meta-analysis. In contrast, GeneWeaver supports this data diversity through combinatorial integration of heterogeneous data, enabling cross-species, cross-platform comparison of multiple experiments. GeneWeaver currently supports data sets from nine species (Mus musculus, Homo sapiens, Rattus norvegicus, Danio rerio, Drosophila melanogaster, Macaca mulatta, Caenorhabditis elegans, Saccharomyces cerevisiae, and Gallus gallus). Many applications are made possible through the representation of gene sets as graphs (Fig. 2). The system provides a database of curated and user-submitted sets of genes defined through a variety of processes, including differential expression experiments, QTL positional candidates, genes annotated to mutant phenotypes, global analysis of gene annotations to MEDLINE abstracts, genetic mapping, and numerous other approaches. Many gene sets are obtained from functional annotations in model organism databases, including the Mouse Genome Database. A system of users, groups, and projects allows for persistent storage or user selections, progressive reanalysis, and result sharing. Although most of these sources and GeneWeaver's current tool suite operate on lists of genes and their association scores ( $p$ value, q-value, correlation coefficient, etc.), an upcoming release will support storage of gene-gene relations to provide for the direct comparison of biological networks.

Each gene set in GeneWeaver is stored with both controlled annotation, free-text description and a curation Tier designation to facilitate retrieval and filtering of relevant studies (Baker et al. 2012). Descriptive meta-content consists of a gene set name, publication information including title and abstract, gene set description which provides detail about the criteria and methods by which genes are assigned to the gene set, a short abbreviation to aid in visualization labels, and annotations to structured vocabularies (Disease Ontology, Open Biomedical Investigations, anatomy, and others) based on automated scan of the text description, and modified through manual curation. Gene sets are denoted by one of five curation tiers (Baker et al. 2012), Tier I designates data derived from curated resources, e.g., Gene Ontology annotations found in model organism databases, Tier II designates data derived from an operation performed on resource data, e.g., genetic correlations to gene expression calculated from GeneNetwork.org, Tier III are gene sets curated from literature or public data submissions that pass curatorial review, Tier IV are public data submissions that have not yet passed curatorial review, and Tier V are data sets uploaded for private use by users and groups.

The structure and contents of the GeneWeaver system have been previously described (Baker et al. 2012), as have the methods for identifier alignment (Jay 2012) and

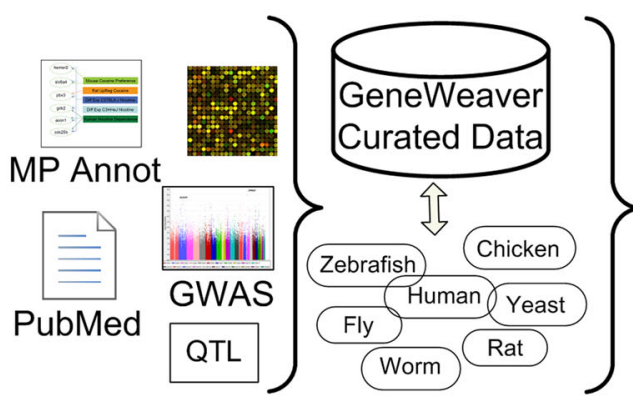

Input Data

Homology mapping

Fig. 1 An overview of the GeneWeaver system. Diverse experimentally derived sets of gene products are curated from the literature, obtained from major public resources, or uploaded by users for shared or private use. These data are harmonized through a graph of gene

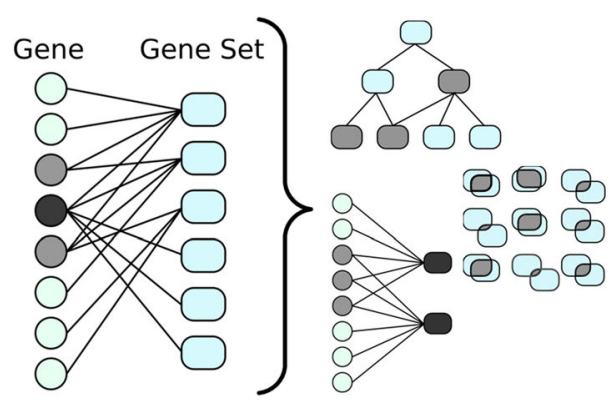

BiGraph Model

identifiers and cross-species homology mappings. The harmonized gene sets are represented as a bipartite graph of genes and gene sets and analyzed using a suite of tools 


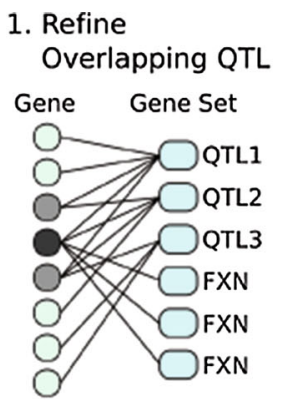

5. Search By Gene

Gene Gene Set

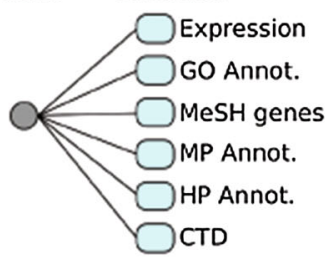

2. Find Highly

Connected Genes

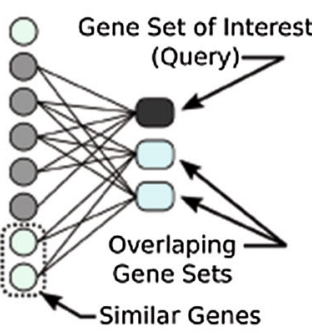

6. Enumerate Intersections

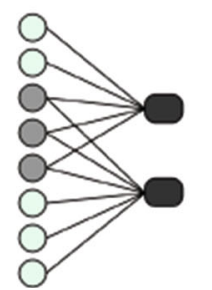

3. Find High Degree

Genes

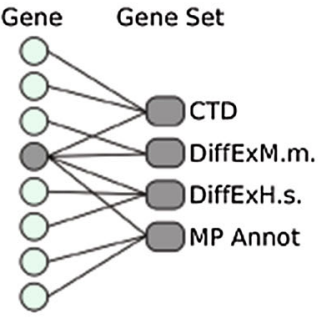

7. Hierarchical Gene Set Similarity

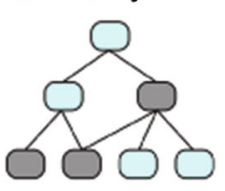

4. Find Similar Gene Sets

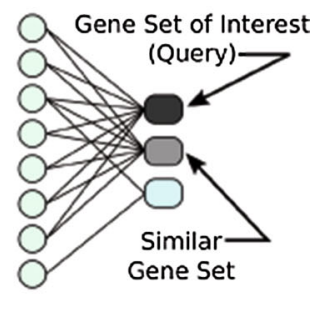

8. Pairwise Gene Set Intersection

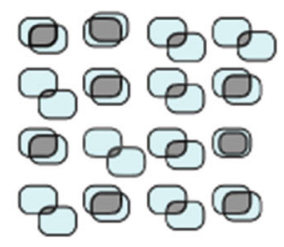

Fig. 2 Biological applications of the GeneWeaver bipartite graph representation. A bipartite graph consists of two partite sets of vertices, with edges between but not within each partite set. A GeneWeaver collection of gene sets consists of one such partite set of vertices representing gene set identifiers and the other partite set representing the genes contained in all the gene sets. Edges between a gene set and genes define gene set membership. This discrete mathematical structure makes possible an efficient application of specialized graph algorithms for rapid comparisons among large numbers of gene sets. (1) To refine overlapping QTL and prioritize functional candidates using trait-relevant data, positional candidates for each locus are entered as gene lists (QTL1-3) and compared to genomic studies of related traits (FXNs). Genes within overlapping QTL are represented in gray, a functionally relevant shared candidate is indicated in black. (2) The ABBA tool is used to find similar genes based on a guilt-by-association-type transitive inference. A gene set of interest is entered into ABBA. Gene sets that overlap the input set either directly or through gene homology among the elements of the sets are retrieved (blue oval nodes). Genes and homologs which are highly similar to the input set based on shared connectivity are retrieved. (3) Highly connected (i.e., high degree) genes are found

combinatorial analysis (Baker et al. 2009, 2012; Zhang et al. 2014). Tutorials for the use of the system are provided at Geneweaver.org/wiki, and the execution steps for a few applications have been described in detail (Jay and Chesler 2014). Here, we highlight some of the biological applications of the GeneWeaver system as employed by users for finding and navigating convergent evidences in diverse functional genomics data.

\section{Comparing experimental results using gene set intersections}

GeneWeaver has several tools that facilitate comparison of studies through set-set overlap among collections of gene sets. Simple pairwise comparisons among a user-selected using the gene set graph tool. A group of gene sets are selected from user uploads or search results. The Gene Set graph represents the most highly connected genes with a user-defined threshold for minimum degree (number of edges) from each gene. The highest degree genes are forced to the right of the plot (although not shown here). (4) To find similar gene sets, users calculate the Jaccard similarity of each gene set in the database to a single user-selected gene set. Results are presented in a ranked table. (5) Entering a single gene identifier in the search box generates a list of gene sets containing the query gene or any homolog or identifier match to the gene. (6) To find the gene elements in the intersection of gene sets, users select a Jaccard similarity value from any table or matrix. (7) The Hierarchical Gene Set Similarity Graph represents successively higher order intersections in a directed acyclic graph, such that individual lists are at the leaves of the graphs and two-way, three-way...n-way intersections are represented on increasingly higher levels of the graph. Shading represents nodes that contain members of a user-selected set of 'emphasis genes.' (8) Pairwise gene set intersections are analyzed using the 'Jaccard similarity' or 'Hypergeometric test' tools. The positive matches (intersection) are compared to the set of possible matches for each pair of gene sets (Color figure online)

or user-submitted collection of gene sets can be made using the Jaccard Similarity tool. More complex intersections can be enumerated through the hierarchical similarity graph and Boolean algebra functions. Using these capabilities, Dever and colleagues retrieved and reanalyzed gene expression data from NCBI's Gene Expression Omnibus system GEO (GSE35864) and uploaded it to GeneWeaver to identify genes differentially expressed between HIVnegative and HIV encephalitis-positive patients across multiple brain regions, including increased expression of the opioid receptor, MOR-1K (Dever et al. 2014). This indicated a potential mechanism by which morphine use may have exacerbated effects in patients with HIV encephalitis. Another example demonstrates how functional genomic analysis can enable the characterization of poorly understood drug effects, possibly discovering new 
applications to related diseases. GeneWeaver analysis was applied to understand the effects of Fingolimod (FTY720), currently used as a treatment for multiple sclerosis. By uploading their set of genes differentially expressed in brains of mice treated with this drug, the authors compared this set with results from other experiments in GeneWeaver, thus enabling identification of a mechanistic role for this drug in decreased histone acetylation, learning, and memory, and ultimately leading to the suggestion that this drug may be clinically useful in the extinction of aversive memories (Hait et al. 2014).

\section{Searching for novel disease-relevant genes with a conserved role across species}

The aggregation of gene sets reveals conserved, frequently occurring but previously un-annotated genes related to biological concepts reflected by the user queries of the database or other collections of gene sets developed or curated by users around a concept of interest. Many genes on these lists are implicated in multiple studies, but have weak or non-existent evidence from deep biological investigations. For example, a cross-species collection of gene sets related to "alcoholism" revealed a highly connected gene associated with alcohol drinking, alcohol response, and alcoholism in multiple species that has no prior association to alcohol phenotypes in mice through MP annotations or alcoholism in humans via OMIM annotations, whereas known alcohol-related genes are found only in a small subset of the retrieved gene lists (Bubier and Chesler 2012). In another investigation, gene set intersection was used to combine and rank results from multiple microarray studies of alcohol-related gene expression in mouse and differential expression in human alcoholic and control brains. From this cross-species analysis, a novel role was discovered for the gene Clic4, which was verified in vivo as modulating alcohol behaviors in fly, worm, and mouse (Bhandari et al. 2012).

\section{Mapping mouse models onto human disease}

A fundamental challenge in harnessing animals for the study of human disease is to understand and identify precise and relevant models. GeneWeaver aids this process through the comparison of genomic correlates of disease across species. In this approach, no assumptions of face validity are required. Rather, the underlying construct reflected in molecular correlates of the disease are directly matched to find a biologically relevant model. For example, one may start with a list of genes associated with human disease and compare the list to GeneWeaver's database of mouse, rat, fly, yeast, worm, and other genomes to find similar gene sets. Utilizing gene2pubmed data (Maglott et al. 2011), publicly available from NCBI, and Pubmed MeSH associations, we generated publicly available GeneWeaver gene sets associated with each MeSH term. A search for similarity to the set of genes annotated to the MeSH term "alcoholism" (GS128735) resulted in the retrieval of a number of relevant sets, including GS216653, protein biomarkers of alcohol abuse (Torrente et al. 2012), and GS137124, differentially expressed genes in Celf4 mutants (Wagnon et al. 2012). We therefore predict that this mouse model is prone to increased alcohol consumption, a previously undiscovered relationship.

\section{Characterizing gene function through search of GeneWeaver}

The GeneWeaver database of greater than 75,000 gene sets is searchable by gene or terms found in free-text descriptions. This enables users to find gene sets containing a gene of interest and thereby identify potential functional roles of the gene across species or provide additional convergent evidence for the role of the gene in a known process. For example, in a study of the genetics of muscle and fat composition in cattle, Cesar and colleagues (Cesar et al. 2014) identified LOXL2 as a positional candidate within a QTL and used the GeneWeaver system to determine that it is also within the positional candidates set for the related phenotype, lean body mass, mapped in Mus musculus QTL, Lbm10 (GS136088), originally reported by Masinde et al. (Masinde et al. 2002).

A single gene query of the system is perhaps most compelling when unknown or poorly characterized genes are evaluated. These so-called "functionally enigmatic genes" can be interrogated to identify patterns among the gene sets in which they appear. For example, the brain ignorome (Pandey et al. 2014), represented in GeneWeaver gene sets GS218259 and GS218282, can be interrogated gene-by-gene to identify stored gene sets that may indicate putative functional roles of the query gene in biological processes. Such analysis can also reveal subtle disease-associated concepts represented in specific experimental data. A search for one such gene (2900011008Rik) reveals that it is worthy of study in addiction-related behaviors (Fig. 3). It is in the QTL for multiple addiction-related traits, including nicotine sensitivity, GS84292 (Gill and Boyle 2005), methamphetamine-induced home cage activity, GS94293 (Grisel et al. 1997), acute functional tolerance to alcohol, GS135263 (Bennett et al. 2007), locomotor activity, GS136175 (Kelly et al. 2003), and nicotine-induced locomotor activity, GS136334 (Boyle and Gill 2009). It is a gene expression correlate of alcohol-induced locomotor activation (GS33885), morphine-induced Ptosis (GS35477), 


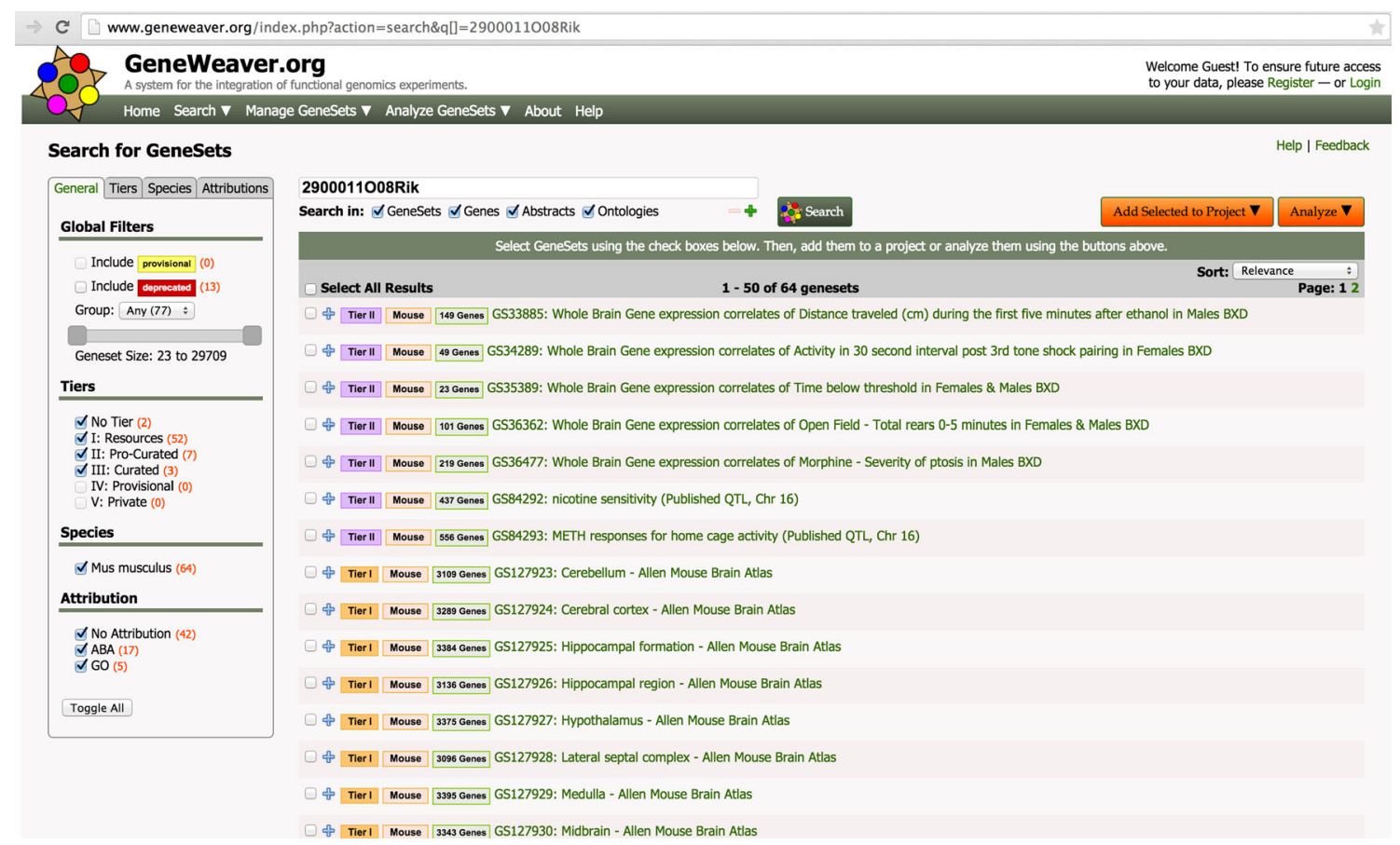

Fig. 3 Search for a single poorly annotated transcript, 2900011O08Rik, results in the retrieval of many gene sets that together reveal experimentally derived insights into its putative role in addiction and other related behaviors

rearing in the open field (GS36362) in BXD recombinant inbred mice (Philip et al. 2010), and haloperidol-induced catalepsy (GS216896) from a study of selected lines (Iancu et al. 2012). It is expressed in multiple brain regions, including the striatum (GS127938), thalamus (GS127939), amygdala (GS127937), pons (GS127933), pallidum (GS127932), midbrain (GS127930), hippocampus (GS129926), cerebellum (GS127923), cerebral cortex (GS127924), and others (Lein et al. 2007). It is also a positional candidate for thalamus volume, gray matter volume, and water maze search time (GS129085) (Dong et al. 2007). The IMPC collection of deletion knock-outs (Koscielny et al. 2014) provides a readily accessible resource in which to evaluate the role of this gene in functional studies, and the information gleaned from aggregate functional genomics evidence in GeneWeaver suggests specific addiction-related phenotypes for evaluation.

In another application of gene-by-gene search, GeneWeaver was used to obtain additional functional information for a list of gene expression correlates of the duration of grooming behavior in mice. In an effort to determine which neural processes are associated with grooming, a behavior which the authors assert may be related to human anxiety disorders, Roth and colleagues (Roth et al. 2013) searched for gene sets containing each individual gene on their list of correlates that had a known human homolog. Using this approach, they were able to find 27 of the 31 correlated genes in gene sets related to mouse neural phenotypes, thereby refining a large list of correlates to those most functionally relevant. These genes were analyzed further in other bioinformatics tools resulting in discovery of a single cross-species interactome network that represents promising translational targets to study grooming behavior.

\section{Evaluating the role of specific genes and diseases through search of GeneWeaver}

By refining the gene-centric query with a functional term, the putative role of a gene in specific traits can be evaluated. For example, in an analysis of the role of voltagegated potassium channels in alcoholism (Padula et al. 2015), GeneWeaver was queried for 'Kcnn1-3 AND alcohol' plus 'Kcnn1-3 AND drug' resulting in retrieval of 26 gene sets from 16 different publications, including many behaviors related to alcohol, nicotine, and illicit drugs. Further analysis of these gene sets using the Gene Set Graph function enabled the identification of 8 other genes that were present in $70 \%$ of the retrieved results and are therefore likely to function with Kcnn 3 in addiction-related phenotypes.

\section{Finding functionally related genes through a gene set graph walk}

A "guilt-by-association" analysis of gene function is formalized in GeneWeaver's Anchored Bicliques of Biomolecular Associations (ABBA) tool, which performs a graph walk that starts from a known gene or gene set and 
transitions to similar genes through shared phenotype connectivity. Currently, this tool uses an unweighted graph, but future releases are planned to make use of weighted graph walks. In its current implementation, the query consists of a gene set of interest. In the first step, a search is performed to find any gene sets that contain a user-specified minimum overlap with genes in the query list. In the second step, ABBA then provides a ranked list of genes based on their prevalence in the resulting gene sets that are not found in the initial list. The resulting genes have similar gene set connectivity with the original input set, but may not have been previously considered in relation to the biological concept represented by the input gene set. Using ABBA, Chan et al. was able to find the genes that were most often found in association with their gene of interest, Clic4. They identified 157 genes which were taken forward in their analysis to understand the role of the Clic4 network in ethanol sensitivity and tolerance (Chan 2013).

Another application of the ABBA tool is in targeted literature curation based on gene similarity to existing disease-associated genes. In one such example, an ABBA search was seeded with genes annotated to autism-like phenotypes in mouse models. The most frequently occurring new genes from gene sets containing multiple input genes were used by MGI curators in a targeted literature search for relevance to autism, resulting in annotation of two additional mutant mouse alleles of Unc5c and Plcb4 which were annotated to autism-related phenotypes in the MGI database (Meehan et al. 2011).

The ABBA tool can also be used to augment information found in collections of GeneWeaver gene sets. For example, a time series analysis of gene expression in the cerebellum of developing mouse Pax6 and Atoh1 mutant mice and their wild-type controls was deposited in GeneWeaver (Ha et al. 2012). A derived gene set formed using the Boolean tool, GS222606, contains 21 genes found in the overlap of the two mouse mutant expression studies. Analysis of this set as an input to the ABBA tool resulted in the retrieval of 483 gene sets containing at least 10 of the input genes and identification of six additional genes that are highly prevalent among the retrieved gene sets. A review of phenotypic alleles of these genes for 'abnormal brain morphology' in Mouse Genome Database reveals that several already have a known role in cerebellar development: Vldlr (Trommsdorff et al. 1999), Ntrk2 (Minichiello et al. 1998), Vegfa (Ferrara et al. 1996), and Rora (Sidman et al. 1962). Two additional genes, Camk2b and Apoe, to date have not been connected to murine cerebellar development in the literature and should be evaluated experimentally. Refinement of QTL positional candidates through
integration of heterogeneous functional evidence

Several other studies have used GeneWeaver to refine QTL positional candidates. This has been done for alcohol-related traits in conventional F2 hybrid crosses (Chesler et al. 2012) and more complex populations including a nociception study in the Diversity Outbred (Recla et al. 2014). In one study, regional gene expression data derived from the Allen Brain Atlas were integrated with QTL positional candidates to identify plausible genetic loci that may regulate the volume of the lateral septal nucleus and associated behaviors (Talishinsky and Rosen 2012).

GeneWeaver provides straightforward integration and resolution of genetic mapping studies of related phenotypes across multiple populations through cross-species synteny and overlap of loci with distinct patterns of allelic variation across strains and across populations. This provides genetic refinement of the often large loci associated with complex traits. Although the concept of multiple-cross QTL comparison is not new (Hitzemann et al. 2002, 2008 ; Li et al. 2011), assembling the resources, aligning genetic loci, and refining sets of variants is cumbersome and requires significant effort or advance planning. By integrating the Mouse Genome Database, Rat Genome Database, and other curated repositories of genetic loci into GeneWeaver, rapid retrieval and cross-trait, cross-population, and crossspecies comparison has been made possible. For example, Mulholland and colleagues were able to compare overlapping mouse QTLs from multiple crosses to identify putative variants in $K c n q 2$ and their potential role in independently mapped alcohol-related phenotypes (McGuier et al., in press).

Comparison of strain distributions across multiple mouse crosses, particularly with some overlap in founders, can even enable refinement to the level of single-nucleotide polymorphisms. For example, a recent study (Bubier et al. 2014) demonstrates the use of GeneWeaver in combination with additional precision genetic resources and genomic databases to identify the precise single-nucleotide polymorphism conferring variation in epigenetic regulation of gene expression as a mechanism for genetic variation in two related biobehavioral processes, alcohol preference and withdrawal. In the first stage of this work, sets of genes related to each function were retrieved and combined to find highly connected preference- and withdrawal-associated genes. In the second stage, these were intersected to reveal a single candidate, $A p 3 m 2$, which was then subject to specific database interrogation and other tests of genetic and functional validity, ultimately enabling us to identify a differentially methylated SNP underlying the observed QTLs. Notably, this example illustrates how very large, 
unresolved legacy results can be integrated and refined to reveal molecular mechanisms for complex traits. Many other sets of highly overlapping loci for related traits can be found in GeneWeaver, facilitating application of this approach to a wide variety of diseases.

\section{Sharing and augmentation of experimentally derived functional genomics data}

The GeneWeaver system allows users to upload and share genome-wide outcomes of experimentation, in contrast to conventional archives for storage of raw experimental data. Sharing and archiving experimental results allows users to readily examine relationships among genome-wide outcomes from very large collections of studies without the need to retrieve and reanalyze each individual study. For example, in a study of the molecular mechanisms of hybrid sterility, Bhattacharyya and colleagues (Bhattacharyya et al. 2013) submitted microarray data to NCBI's Gene Expression Omnibus system (GSE41707) to make raw data available. They also self-archived four enriched gene sets (GeneWeaver GS213073-GS213077) specifically reflecting expression differences among sterile hybrids and fertile controls. Each set represents the comparison of cross founders and F1 progeny.

By placing these data in GeneWeaver, additional analyses are readily possible. For example, a hierarchical intersection analysis reveals 471 genes found on all four lists. This new list was used to query the database for similar gene sets based on Jaccard similarity to all database contents. This search revealed overlap of the differentially expressed genes with positional candidates within a QTL for testes weight, GS136884 (Zidek et al. 1998), and GO annotations to pheromone receptor activity and response (GS180456, GS1192092). Eleven of these gene sets were selected and placed in a GeneWeaver project. The original intersection sets were specified as 'emphasis genes' to highlight them in results output. A hierarchical similarity analysis and gene set graph analysis reveals that Vmn1r199 and other pheromone receptors are highly connected genes, suggesting that one candidate mechanism of infertility is disruption in pheromone signaling and its potential effects on reproductive behavior. An additional overlapping subnetwork includes two reproductive QTLs mapping to the M.musculus Chromosome X, containing positional candidates Spin2a,Diap2, and Taf7 l. Of these genes, Diap2 has been associated with female sterility (Bione et al. 1998) and $T a f 7 l$ has been associated with male sterility (Akinloye et al. 2007; Stouffs et al. 2006). Because these QTLs were mapped in multiple crosses, it may be possible to identify candidate genetic variants in the Sanger Mouse Genomes sequence resource (Keane et al. 2011) to further refine the causal variants in these loci. Furthermore, the identification of overlapping and functionally relevant gene sets provides insight into additional biological endpoints, beyond sterility, that may be influenced by such variants.

\section{Data-driven disease classification}

The aggregate analysis of heterogeneous data from diverse, genome-wide genomic associates of complex disease provides a means of data-driven classification of disease based on an overlapping biological substrate. To demonstrate the application of this strategy on which we have previously elaborated (Chesler and Logan 2012), we used GeneWeaver's hierarchical similarity graph to partition gene annotation sets for psychiatric disorders. MEDLINE abstracts containing gene symbols and their annotations to $\mathrm{MeSH}$ terms were used to construct sets of genes associated with each term in the Psychiatric disorder MeSH subtree. Analysis in the hierarchical similarity graph tool revealed genes related to a constellation of psychiatric disorders. The tool subdivided the disorder based on 18 genes associated with 'Anxiety' but not 'Depression with alcohol use' and two associated with 'Depression with alcohol use' and not 'Anxiety.' Evaluation of Oxtr and $A b c b 1$ genes in anxiety and alcohol assays, along with representatives of the 18 anxiety genes not associated with depression and alcohol dependence, will test the validity of this classification. One caveat to note is that in an effort to reduce artifacts, genes were only associated to a particular MeSH term if they occurred in a minimum of two publications associated to that MeSH term. As a result, GeneWeaver may find partitions based on missing or weak connectivity, i.e., absence of evidence, not evidence of absence. The precision and accuracy of this strategy should be greatly improved over time with the inclusion of larger collections of data.

\section{Interpreting and comparing gene co-expression networks}

In most of the examples we have provided, sets of genes are compared and contrasted. This gene set integration provides a versatile approach to dealing with most functional genomics result types, but increasingly network analysis methods provide even greater precision. Many of the technologies used to generate lists of biologically important genes are error prone. The more stringent criteria provided by gene-gene correlation or interaction provide greater evidence of a common role of genes in a process. Gene co-expression network analysis is widely employed to find modules of genes that behave in concert in relation 
to biological manipulations or across segregating populations. Although these are often represented as lists or sets of module members, they implicitly reflect high interconnectivity among the members. We have previously shown that dense co-expression sub-networks have high biological relevance, and the interpretation of individual subgraphs can reveal functional roles of these modules.

Gene co-expression modules from a study of haloperidol-induced catalepsy in short-term selected mouse lines (Iancu et al. 2012) were entered into GeneWeaver. The functional roles of these modules can be characterized by a search for similar gene sets. For example, one co-expression cluster, GS216879, was compared to the rest of the database. The five most similar gene sets were GS35275, striatal gene expression correlates of acoustic startle (Philip et al. 2010), GS122992, nitrosobenzylmethylamine-interacting genes from the Comparative Toxicogenomics Database (Davis et al. 2015), GS33995, correlates of blood alcohol concentration in BXD mice (Philip et al. 2010), and GS1778, differential expression in mice with high and low acute functional tolerance to alcohol (Tabakoff et al. 2003). Genes common to four of these five sets, Timm $8 A$ and Gng5, are revealed using GeneWeaver's gene set graph tool. Other alcohol-related co-expression network studies have been placed in GeneWeaver, including co-expression of genes in BXD Recombinant inbred strains with predisposition to alcohol consumption (Vanderlinden et al. 2013).

We have recently developed a strategy to extrapolate GeneWeaver's gene set integration tools to the comparison of network edge relations across collections of graphs (Baker et al. 2014). This will ultimately enable a direct comparison of biological networks not just through the sharing of molecular members, but also through the consistency of interactions among those molecules. Network similarity graphs will permit researchers to evaluate the presence and conservation of co-expression, molecular pathways, and molecular interaction networks across biological perturbations, genetic populations, and other heterogeneous contexts.

\section{Summary: finding consilience among heterogeneous experiments}

Phenomenal curated biological data resources provide a rich context in which to explore functional genomics experiments. By placing the data from multiple curated databases into a single analytic framework and populating it with empirical experimental outcomes from genomewide experimentation, data can be efficiently combined and navigated to find consilience among heterogeneous experiments. Many paths to inquiry are enabled by the system, either through the extension of a set of results or the refinement from a collection of studies. By minimizing data integration hurdles and providing a suite of set operations, users can combine and compare gene sets across experiments and species to find convergence of evidence across experiment types, model systems, organisms, and diseases.

As the highlighted examples demonstrate, the insights gained from this exploration may either be expansive, enabling users to extend existing findings by effectively navigating overlapping experimental results, or refining, reducing a wealth of genes from a single experimental result to a subset of highly supported genes across experiments. In both cases, the availability of deep and broad evidence from many sources frees the biological question from a single, restrictive context. It also provides independent, orthogonal approaches to the research question, free from the biases inherent in single measurements of a biological or behavioral concept, specific characteristics of populations including linkage disequilibrium, distributions of variants, and the presence of particular gene products in various species.

There has been a recent concern about the ability to replicate and reproduce findings in model organism studies (Seok et al. 2013; Vasilevsky et al. 2013). Reproducing experiments and replicating their results is remarkably challenging in the face of variation and limited documentation of experimental systems including model organisms, test procedures and parameters, and testing and housing environments. Resources like the Mouse Phenome Database (Grubb et al. 2014) and the International Mouse Phenotyping Consortium's mousephenotypes.org (Koscielny et al. 2014) which follows ARRIVE guidelines (Kilkenny et al. 2010) for reporting experimental methods, provide deep insight into how data are collected in hopes that investigators will have an improved ability to reproduce each other's experiments. Although replication of experiments in a logically consistent and falsifiable manner improves confidence in results and has been a backbone of the scientific method, the emphasis on strict replication is questionable, as the replication may be perfect; yet the generalizability of the experimental result to the research question at hand may be quite limited. This is particularly the case when exact replication of experimental conditions is nearly impossible to obtain, as in the case of laboratory environmental variation. If the objective is to test a hypothesis concerning relations among treatment conditions, environmental perturbations, and organismal variables including sex and genetic variation in a manner that translates down the hall, across the world, or to another species, one might rather seek convergence of evidence across experiments. 
Seeking 'consilience' - a common result regardless of how the question is asked or how the phenotype is assayed-may be more valuable than simply striving to replicate a system containing many unknown and poorly understood environmental and experimental variables. The main challenge is in the integration and interrogation of diverse data. Functional genomics experimentation has been described as purely inductive or 'hypothesis generating.' But these techniques are not merely inductive; they provide a quantitation of the state of biological systems in many contexts. Classification of the conditions under which particular results are observed provides insight into the relations among those conditions, and these relations can be subject to quantitative evaluation. GeneWeaver was designed to support the search for consilience among genetic and genomic studies of disease through the convergence of evidence in genome-wide functional genomics experiments. It provides analytic approaches to aggregate, integrate, and classify many heterogeneous experimental findings, so that we may interpret, rather than merely reject, variation in results obtained across independent studies. To quote William Whewell, "the evidence in favour of our induction is of a much higher and more forcible character when it enables us to explain and determine cases of a kind different from those which were contemplated in the formation of our hypothesis...No accident could give rise to such an extraordinary coincidence" (Whewell 1847).

Acknowledgments The original development of the GeneWeaver system was supported by U01AA13499 and U24AA13513. It is currently supported by NIH R01 AA18776, jointly funded by NIAAA and NIDA. Data were obtained from many public resources made available for academic use.

Open Access This article is distributed under the terms of the Creative Commons Attribution 4.0 International License (http://creativecommons.org/licenses/by/4.0/), which permits unrestricted use, distribution, and reproduction in any medium, provided you give appropriate credit to the original author(s) and the source, provide a link to the Creative Commons license, and indicate if changes were made.

\section{References}

Akinloye O, Gromoll J, Callies C, Nieschlag E, Simoni M (2007) Mutation analysis of the X-chromosome linked, testis-specific TAF7L gene in spermatogenic failure. Andrologia 39:190-195

Baker EJ, Jay JJ, Philip VM, Zhang Y, Li Z, Kirova R, Langston MA, Chesler EJ (2009) Ontological discovery environment: a system for integrating gene-phenotype associations. Genomics 94:377-387

Baker EJ, Jay JJ, Bubier JA, Langston MA, Chesler EJ (2012) GeneWeaver: a web-based system for integrative functional genomics. Nucleic Acids Res 40:D1067-D1076

Baker E, Culpepper C, Philips C, Bubier J, Langston M, Chesler E (2014) Identifying common components across biological network graphs using a bipartite data model. BMC Proc 8:S4
Bennett B, Downing C, Carosone-Link P, Ponicsan H, Ruf C, Johnson TE (2007) Quantitative trait locus mapping for acute functional tolerance to ethanol in the $\mathrm{L} \times \mathrm{S}$ recombinant inbred panel. Alcohol Clin Exp Res 31:200-208

Bhandari P, Hill JS, Farris SP, Costin B, Martin I, Chan CL, Alaimo JT, Bettinger JC, Davies AG, Miles MF, Grotewiel M (2012) Chloride intracellular channels modulate acute ethanol behaviors in Drosophila, Caenorhabditis elegans and mice. Genes Brain Behav 11:387-397

Bhattacharyya T, Gregorova S, Mihola O, Anger M, Sebestova J, Denny P, Simecek P, Forejt J (2013) Mechanistic basis of infertility of mouse intersubspecific hybrids. Proc Natl Acad Sci U S A 110:E468-E477

Bione S, Sala C, Manzini C, Arrigo G, Zuffardi O, Banfi S, Borsani G, Jonveaux P, Philippe C, Zuccotti M, Ballabio A, Toniolo D (1998) A human homologue of the Drosophila melanogaster diaphanous gene is disrupted in a patient with premature ovarian failure: evidence for conserved function in oogenesis and implications for human sterility. Am J Hum Genet 62:533-541

Boyle AE, Gill KJ (2009) Genetic analysis of the psychostimulant effects of nicotine in chromosome substitution strains and F2 crosses derived from $\mathrm{A} / \mathrm{J}$ and $\mathrm{C} 57 \mathrm{BL} / 6 \mathrm{~J}$ progenitors. Mamm Genome 20:34-42

Bubier JA, Chesler EJ (2012) Accelerating discovery for complex neurological and behavioral disorders through systems genetics and integrative genomics in the laboratory mouse. Neurotherapeutics 9:338-348

Bubier JA, Jay JJ, Baker CL, Bergeson SE, Ohno H, Metten P, Crabbe JC, Chesler EJ (2014) Identification of a QTL in Mus musculus for alcohol preference, withdrawal, and Ap3m2 expression using integrative functional genomics and precision genetics. Genetics 197:1377-1393

Cesar AS, Regitano LC, Mourao GB, Tullio RR, Lanna DP, Nassu RT, Mudado MA, Oliveira PS, do Nascimento ML, Chaves AS, Alencar MM, Sonstegard TS, Garrick DJ, Reecy JM, Coutinho LL (2014) Genome-wide association study for intramuscular fat deposition and composition in Nellore cattle. BMC Genet 15:39

Chan RF (2013) Genetic Analysis of Ethanol Sensitivity and Tolerance in Drosophila. Virginia Commonwealth University Richmond, Virginia

Chesler EJ, Logan RW (2012) Opportunities for bioinformatics in the classification of behavior and psychiatric disorders. Int Rev Neurobiol 104:183-211

Chesler EJ, Plitt A, Fisher D, Hurd B, Lederle L, Bubier JA, Kiselycznyk C, Holmes A (2012) Quantitative trait loci for sensitivity to ethanol intoxication in a C57BL/6Jx129S1/SvImJ inbred mouse cross. Mamm Genome 23:305-321

Davis AP, Grondin CJ, Lennon-Hopkins K, Saraceni-Richards C, Sciaky D, King BL, Wiegers TC, Mattingly CJ (2015) The Comparative Toxicogenomics Database's 10th year anniversary: update 2015. Nucleic Acids Res 43:D914-D920

Dever SM, Costin BN, Xu R, El-Hage N, Balinang J, Samoshkin A, O'Brien MA, McRae M, Diatchenko L, Knapp PE, Hauser KF (2014) Differential expression of the alternatively spliced OPRM1 isoform mu-opioid receptor-1 K in HIV-infected individuals. Aids 28:19-30

Dong H, Martin MV, Colvin J, Ali Z, Wang L, Lu L, Williams RW, Rosen GD, Csernansky JG, Cheverud JM (2007) Quantitative trait loci linked to thalamus and cortex gray matter volumes in BXD recombinant inbred mice. Heredity (Edinb) 99:62-69

Ferrara N, Carver-Moore K, Chen H, Dowd M, Lu L, O'Shea KS, Powell-Braxton L, Hillan KJ, Moore MW (1996) Heterozygous embryonic lethality induced by targeted inactivation of the VEGF gene. Nature 380:439-442 
Gill KJ, Boyle AE (2005) Genetic basis for the psychostimulant effects of nicotine: a quantitative trait locus analysis in $\mathrm{AcB} / \mathrm{BcA}$ recombinant congenic mice. Genes Brain Behav 4:401-411

Grisel JE, Belknap JK, O’Toole LA, Helms ML, Wenger CD, Crabbe JC (1997) Quantitative trait loci affecting methamphetamine responses in BXD recombinant inbred mouse strains. J Neurosci 17:745-754

Grubb SC, Bult CJ, Bogue MA (2014) Mouse phenome database. Nucleic Acids Res 42:D825-D834

Ha TJ, Swanson DJ, Kirova R, Yeung J, Choi K, Tong Y, Chesler EJ, Goldowitz D (2012) Genome-wide microarray comparison reveals downstream genes of Pax6 in the developing mouse cerebellum. Eur J Neurosci 36:2888-2898

Hait NC, Wise LE, Allegood JC, O'Brien M, Avni D, Reeves TM, Knapp PE, Lu J, Luo C, Miles MF, Milstien S, Lichtman AH, Spiegel S (2014) Active, phosphorylated fingolimod inhibits histone deacetylases and facilitates fear extinction memory. Nat Neurosci 17:971-980

Hitzemann R, Malmanger B, Cooper S, Coulombe S, Reed C, Demarest K, Koyner J, Cipp L, Flint J, Talbot C, Rademacher B, Buck K, McCaughran J Jr (2002) Multiple cross mapping (MCM) markedly improves the localization of a QTL for ethanol-induced activation. Genes Brain Behav 1:214-222

Hitzemann R, Belknap JK, McWeeney SK (2008) Quantitative trait locus analysis: multiple cross and heterogeneous stock mapping. Alcohol Res Health 31:261-265

Iancu OD, Darakjian P, Malmanger B, Walter NA, McWeeney S, Hitzemann R (2012) Gene networks and haloperidol-induced catalepsy. Genes Brain Behav 11:29-37

Jay JJ (2012) Cross species integration of functional genomics experiments. Int Rev Neurobio 104:1-24

Jay JJ, Chesler EJ (2014) Performing integrative functional genomics analysis in GeneWeaver.org. Methods Mol Biol 1101:13-29

Kang EY, Han B, Furlotte N, Joo JW, Shih D, Davis RC, Lusis AJ, Eskin E (2014) Meta-analysis identifies gene-by-environment interactions as demonstrated in a study of 4,965 mice. PLoS Genet 10:e1004022

Keane TM, Goodstadt L, Danecek P, White MA, Wong K, Yalcin B, Heger A, Agam A, Slater G, Goodson M, Furlotte NA, Eskin E, Nellaker C, Whitley H, Cleak J, Janowitz D, Hernandez-Pliego P, Edwards A, Belgard TG, Oliver PL, McIntyre RE, Bhomra A, Nicod J, Gan X, Yuan W, van der Weyden L, Steward CA, Bala S, Stalker J, Mott R, Durbin R, Jackson IJ, Czechanski A, Guerra-Assuncao JA, Donahue LR, Reinholdt LG, Payseur BA, Ponting CP, Birney E, Flint J, Adams DJ (2011) Mouse genomic variation and its effect on phenotypes and gene regulation. Nature 477:289-294

Kelly MA, Low MJ, Phillips TJ, Wakeland EK, Yanagisawa M (2003) The mapping of quantitative trait loci underlying strain differences in locomotor activity between 129S6 and C57BL/6 J mice. Mamm Genome 14:692-702

Kilkenny C, Browne WJ, Cuthill IC, Emerson M, Altman DG (2010) Improving bioscience research reporting: the ARRIVE guidelines for reporting animal research. PLoS Biol 8:e1000412

Koscielny G, Yaikhom G, Iyer V, Meehan TF, Morgan H, AtienzaHerrero J, Blake A, Chen CK, Easty R, Di Fenza A, Fiegel T, Grifiths M, Horne A, Karp NA, Kurbatova N, Mason JC, Matthews P, Oakley DJ, Qazi A, Regnart J, Retha A, Santos LA, Sneddon DJ, Warren J, Westerberg H, Wilson RJ, Melvin DG, Smedley D, Brown SD, Flicek P, Skarnes WC, Mallon AM, Parkinson H (2014) The international mouse phenotyping consortium web portal, a unified point of access for knockout mice and related phenotyping data. Nucleic Acids Res 42:D802D809

Lein ES, Hawrylycz MJ, Ao N, Ayres M, Bensinger A, Bernard A, Boe AF, Boguski MS, Brockway KS, Byrnes EJ, Chen L, Chen
TM, Chin MC, Chong J, Crook BE, Czaplinska A, Dang CN, Datta S, Dee NR, Desaki AL, Desta T, Diep E, Dolbeare TA, Donelan MJ, Dong HW, Dougherty JG, Duncan BJ, Ebbert AJ, Eichele G, Estin LK, Faber C, Facer BA, Fields R, Fischer SR, Fliss TP, Frensley C, Gates SN, Glattfelder KJ, Halverson KR, Hart MR, Hohmann JG, Howell MP, Jeung DP, Johnson RA, Karr PT, Kawal R, Kidney JM, Knapik RH, Kuan CL, Lake JH, Laramee AR, Larsen KD, Lau C, Lemon TA, Liang AJ, Liu Y, Luong LT, Michaels J, Morgan JJ, Morgan RJ, Mortrud MT, Mosqueda NF, Ng LL, Ng R, Orta GJ, Overly CC, Pak TH, Parry SE, Pathak SD, Pearson OC, Puchalski RB, Riley ZL, Rockett HR, Rowland SA, Royall JJ, Ruiz MJ, Sarno NR, Schaffnit K, Shapovalova NV, Sivisay T, Slaughterbeck CR, Smith SC, Smith KA, Smith BI, Sodt AJ, Stewart NN, Stumpf KR, Sunkin SM, Sutram M, Tam A, Teemer CD, Thaller C, Thompson CL, Varnam LR, Visel A, Whitlock RM, Wohnoutka PE, Wolkey CK, Wong VY, Wood M, Yaylaoglu MB, Young RC, Youngstrom BL, Yuan XF, Zhang B, Zwingman TA, Jones AR (2007) Genome-wide atlas of gene expression in the adult mouse brain. Nature 445:168-176

Li H, Bradbury P, Ersoz E, Buckler ES, Wang J (2011) Joint QTL linkage mapping for multiple-cross mating design sharing one common parent. PLoS ONE 6:e17573

Maglott D, Ostell J, Pruitt KD, Tatusova T (2011) Entrez gene: genecentered information at NCBI. Nucleic Acids Res 39:D52-D57

Masinde GL, Li X, Gu W, Davidson H, Hamilton-Ulland M, Wergedal J, Mohan S, Baylink DJ (2002) Quantitative trait loci (QTL) for lean body mass and body length in MRL/MPJ and SJL/J F(2) mice. Funct Integr Genomics 2:98-104

McGuier NS, Griffin WC, Padula AE, Chesler E, Mulholland PJ (in press) Kv7 channels in the nucleus accumbens are altered by chronic drinking and are targets for reducing alcohol consumption. Addict Biol

Meehan TF, Carr CJ, Jay JJ, Bult CJ, Chesler EJ, Blake JA (2011) Autism candidate genes via mouse phenomics. J Biomed Inform 44(Suppl 1):S5-S11

Minichiello L, Casagranda F, Tatche RS, Stucky CL, Postigo A, Lewin GR, Davies AM, Klein R (1998) Point mutation in trkB causes loss of NT4-dependent neurons without major effects on diverse BDNF responses. Neuron 21:335-345

Padula AE, Griffin WC 3rd, Lopez MF, Nimitvilai S, Cannady R, McGuier NS, Chesler EJ, Miles MF, Williams RW, Randall PK, Woodward JJ, Becker HC, Mulholland PJ (2015) KCNN genes that encode small-conductance $\mathrm{Ca}(2+)$-activated $\mathrm{K}(+)$ channels influence alcohol and drug addiction. Neuropsychopharmacology 40(8):1928-1939

Pandey AK, Lu L, Wang X, Homayouni R, Williams RW (2014) Functionally enigmatic genes: a case study of the brain ignorome. PLoS ONE 9:e88889

Philip VM, Duvvuru S, Gomero B, Ansah TA, Blaha CD, Cook MN, Hamre KM, Lariviere WR, Matthews DB, Mittleman G, Goldowitz D, Chesler EJ (2010) High-throughput behavioral phenotyping in the expanded panel of BXD recombinant inbred strains. Genes Brain Behav 9:129-159

Recla JM, Robledo RF, Gatti DM, Bult CJ, Churchill GA, Chesler EJ (2014) Precise genetic mapping and integrative bioinformatics in diversity outbred mice reveals hydin as a novel pain gene. Mamm Genome 25:211-222

Roth A, Kyzar EJ, Cachat J, Stewart AM, Green J, Gaikwad S, O'Leary TP, Tabakoff B, Brown RE, Kalueff AV (2013) Potential translational targets revealed by linking mouse grooming behavioral phenotypes to gene expression using public databases. Prog Neuropsychopharmacol Biol Psychiatry 40: 312-325

Seok J, Warren HS, Cuenca AG, Mindrinos MN, Baker HV, Xu W, Richards DR, McDonald-Smith GP, Gao H, Hennessy L, 
Finnerty CC, Lopez CM, Honari S, Moore EE, Minei JP, Cuschieri J, Bankey PE, Johnson JL, Sperry J, Nathens AB, Billiar TR, West MA, Jeschke MG, Klein MB, Gamelli RL, Gibran NS, Brownstein BH, Miller-Graziano C, Calvano SE, Mason PH, Cobb JP, Rahme LG, Lowry SF, Maier RV, Moldawer LL, Herndon DN, Davis RW, Xiao W, Tompkins RG (2013) Genomic responses in mouse models poorly mimic human inflammatory diseases. Proc Natl Acad Sci U S A 110:3507-3512

Sidman RL, Lane PW, Dickie MM (1962) Staggerer, a new mutation in the mouse affecting the cerebellum. Science 137:610-612

Stouffs K, Willems A, Lissens W, Tournaye H, Van Steirteghem A, Liebaers I (2006) The role of the testis-specific gene hTAF7L in the aetiology of male infertility. Mol Hum Reprod 12:263-267

Tabakoff B, Bhave SV, Hoffman PL (2003) Selective breeding, quantitative trait locus analysis, and gene arrays identify candidate genes for complex drug-related behaviors. J Neurosci 23:4491-4498

Talishinsky A, Rosen GD (2012) Systems genetics of the lateral septal nucleus in mouse: heritability, genetic control, and covariation with behavioral and morphological traits. PLoS ONE 7:e44236

Torrente MP, Freeman WM, Vrana KE (2012) Protein biomarkers of alcohol abuse. Expert Rev Proteomics 9:425-436

Trommsdorff M, Gotthardt M, Hiesberger T, Shelton J, Stockinger W, Nimpf J, Hammer RE, Richardson JA, Herz J (1999) Reeler/
Disabled-like disruption of neuronal migration in knockout mice lacking the VLDL receptor and ApoE receptor 2. Cell 97:689-701

Vanderlinden LA, Saba LM, Kechris K, Miles MF, Hoffman PL, Tabakoff B (2013) Whole brain and brain regional coexpression network interactions associated with predisposition to alcohol consumption. PLoS ONE 8:e68878

Vasilevsky NA, Brush MH, Paddock H, Ponting L, Tripathy SJ, Larocca GM, Haendel MA (2013) On the reproducibility of science: unique identification of research resources in the biomedical literature. PeerJ 1:e148

Wagnon JL, Briese M, Sun W, Mahaffey CL, Curk T, Rot G, Ule J, Frankel WN (2012) CELF4 regulates translation and local abundance of a vast set of mRNAs, including genes associated with regulation of synaptic function. PLoS Genet 8:e1003067

Whewell W (1847) The Philosophy of the inductive sciences, founded upon their history, 2nd edn. John W. Parker, London

Zhang Y, Phillips CA, Rogers GL, Baker EJ, Chesler EJ, Langston MA (2014) On finding bicliques in bipartite graphs: a novel algorithm and its application to the integration of diverse biological data types. BMC Bioinform 15:110

Zidek V, Musilova A, Pintir J, Simakova M, Pravenec M (1998) Genetic dissection of testicular weight in the mouse with the BXD recombinant inbred strains. Mamm Genome 9:503-505 